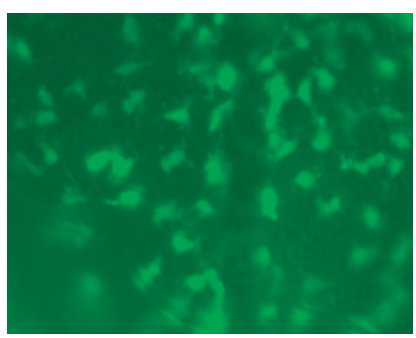

p 432

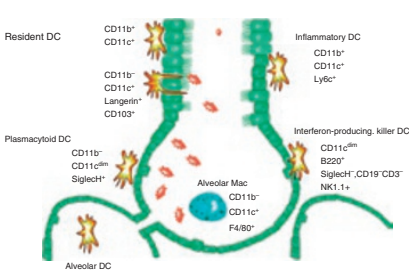

p 442

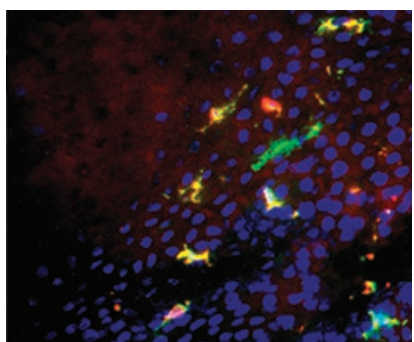

p 451

\section{Food for thought}

Food allergies are on the rise. In this issue's News \& Highlights section Dale Umetsu comments on progress and gaps in understanding the etiology, prevention, and treatment of this important clinical problem. See page 422

\section{Development of dendritic cell subsets}

Priyanka Sathe and Ken Shortman review the abundance of literature that addresses the development of dendritic cell subsets. The authors underscore areas of conflicting information and highlight issues that call for further research. See page 425

\section{Just how similar are DCs and macrophages?}

In this review, David Hume puts forth the notions that we have put too much emphasis on documenting differences in cell surface phenotypes of monocytes, macrophages, and dendritic cells, and that it is now time to think about describing these cells collectively as cells of the mononuclear phagocyte system. See page 432

\section{Upsetting the balance of lung dendritic cells}

Infections and inflammatory conditions can alter the balance of dendritic cell subsets. Corine GeurtsvanKessel and Bart Lambrecht focus their attention on the lung and examine the impact of upsetting the homeostatic balance among various DC subsets. See page 442

\section{Dendritic cells and} macrophages in the GU tract

In this review, Norifumi lijima and colleagues discuss the lineage differences and functional diversity of the various antigen-presenting-cell subsets that line the genitourinary tract. They emphasize the disparate requirements for these cells in maintaining defense against sexually transmitted infections while retaining the ability to exhibit tolerance to a fetus.

See page 451

\section{Digesting intestinal dendritic cells and macrophage function}

The past year has seen a dramatic explosion in discoveries related to the function of intestinal dendritic cells and macrophages. In this review, Brian Kelsall collates the wealth of new information into a digestible format. See page 460

\section{Skin accessory cell discoveries}

Skin dendritic cells and macrophages have been difficult to study, particularly in vivo. Mark Udey and Keisuke Nagao review the recent advances in the field and provide commentary on the sometimes surprising new insights. See page 470

\section{The benefits of preserving T cells in HIV}

Two research articles address the importance of understanding the requirement, regulation, and function of $\mathrm{CD} 4^{+} \mathrm{T}$ cells in the gut during HIV infection. Mónica Macal and colleagues demonstrate that even modest (as little as 50\%) $\mathrm{CD} 4{ }^{+} \mathrm{T}$-cell restoration during highly active antiretroviral therapy may be sufficient to restore polyfunctional HIV-specific cellular immune responses. In a related article, Monica Vaccari and colleagues show that even though a protective DNA-poxvirus-based vaccine against SIV does not prevent acute loss of $\mathrm{CD} 4^{+} \mathrm{T}$ cells from the intestine, it limits viral seeding at mucosal sites and results in enhanced T-cell repletion in the gut over time.

See pages 475 and 497

\section{Improving mucosal vaccines}

Using a large animal model, Janet Wee and colleagues demonstrate that local mucosal delivery of influenza vaccine using deep lung vaccination and ISCOMATRIX adjuvant can drive vigorous antibody responses that translate into improved protection from influenza. Because very low doses of vaccine were required, the authors suggest that lung vaccination may be useful when vaccine supplies are limited, such as may occur during a pandemic.

See page 489 